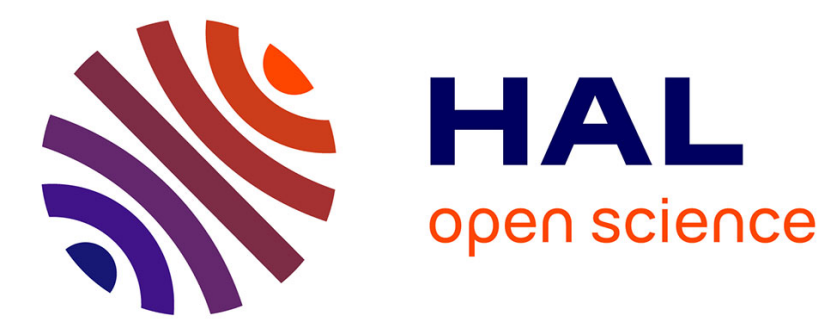

\title{
Terra nigra trouvée à Vichy (Allier)
}

Colette Bémont

\section{To cite this version:}

Colette Bémont. Terra nigra trouvée à Vichy (Allier). Gallia - Fouilles et monuments archéologiques en France métropolitaine, 1972, 30 (1), pp.149-166. 10.3406/galia.1972.2598 . hal-01934883

\section{HAL Id: hal-01934883 \\ https://hal.science/hal-01934883}

Submitted on 11 Mar 2020

HAL is a multi-disciplinary open access archive for the deposit and dissemination of scientific research documents, whether they are published or not. The documents may come from teaching and research institutions in France or abroad, or from public or private research centers.
L'archive ouverte pluridisciplinaire HAL, est destinée au dépôt et à la diffusion de documents scientifiques de niveau recherche, publiés ou non, émanant des établissements d'enseignement et de recherche français ou étrangers, des laboratoires publics ou privés.

\section{(이) $\$$}

Distributed under a Creative Commons Attribution - NonCommercial - NoDerivatives| 4.0 


\title{
TERRA NIGRA TROUVÉE A VICHY (Allier)
}

\author{
par Colette BÉMONT
}

La collection Rambert du Musćc des Antiquités nationales de Saint-Germain-en-Laye contient, à côté d'une abondante série de vases ou de moules sigillés, quelques pièces estampillées de facture plus nettement provinciale et dignes, elles aussi, d'attention. Il s'agit de tessons de céramique fumigée fine qui, comme le reste du legs Rambert, sont tenus pour provenir du sous-sol de Vichy². J'ai signalé déjà ${ }^{2}$ deux fragments de vases appartenant à ce groupe et qui présentent la particularité de concilier un décor intérieur - constitué de zones grises alternativement claires et foncées - et l'usage d'une estampille centrale sur deux lignes, de type gallo-romain ${ }^{3}$. Les neuf autres pièces, tout en s'apparentant à une catégorie plus banale : celle de la poterie fine, grise ou noire, monochrome, ne sont pas dépourvues d'un intérêt certain, bien qu'inégal selon les cas et l'état de conservation des vases. En voici un rapide inventaire.

A-C. : trois tessons (fig. 1, A-C) marqués de la mème estampille centrale : COT $\Theta I O[-] / A$ VOT.[-]. $\Lambda$ (M.A.N. 49571) : fragment d'un fond plat, légèrement bombé au centre, aminci vers la périphérie (épaiss. de $7,5 \mathrm{~mm}$ à $3 \mathrm{~mm}$ ); d'après le fragment conservé $\varnothing$ supérieur à $80 \mathrm{~mm}$; pâte gris clair ; surface intérieure gris sombre soigneusement lissée; surface extérieure plus claire et marquée de la trace en spirale d'un lissage incomplet. B (M.A.X. 49590) : mêmes remarques, dans l'ensemble, que pour le précédent tesson ; bombement moins accentué, épaiss. variant de 6,5 à 5,5 mm ; $\varnothing$ supérieur à $70 \mathrm{~mm}$; couleur superficielle : gris moyen. G (M.A.N. 49579) : mêmes remarques techniques que pour les précédents numéros ; épaiss. à peu près constante et égale à $5 \mathrm{~mm}$ env.; g supérieur à $95 \mathrm{~mm}$; décor : cercle guilloché composé de quatre rangs de stries (larg. $11 \mathrm{~mm} ; \varnothing$ intérieur : $60 \mathrm{~mm}$ ). D)-E : deux fragments (fig. 1, D-E) marqués IITOVA/SIICI[--]. D (M.A.N. 49577) : fond plat bombé au centre ; épaiss. régulière d'environ $7,5 \mathrm{~mm}$; $\varnothing$ supérieur à $90 \mathrm{~mm}$; pâte gris clair ; surface intérieure gris moyen, bien lissée, surface extérieure assez soignée, de mème couleur ; E (M.A.N. 49580) : fond plat, épaissi au centre (épaiss. de $7 \mathrm{~mm}$ à $6 \mathrm{~mm}$ ), à peine bombé ; જ supérieur à $120 \mathrm{~mm}$; pâte gris clair ; surface intérieure gris sombre, surface extérieure : cf. nos $49571,49590,49579$; décor : cercle guilloché à cinq rangs de stries (larg. $14 \mathrm{~mm} ; \varnothing$ intérieur : $90 \mathrm{~mm}$ ). F : fragment d'un petit bol (fig. 2, F) marqué ATI[- - ]/[- - ] (M.A.X. 49582) ; pied bas non rapporté ( $\varnothing$ extérieur :

1 A. Monlet, Graffiles et signalures, dans Tourisme, 1957, n. 3.

2 Vases gris décorés et signés du début du Ier siècle. P. C., dans Antiquités Nationales, 3, 1971, p. 67-78.

3 A.VS/AVOTI. 

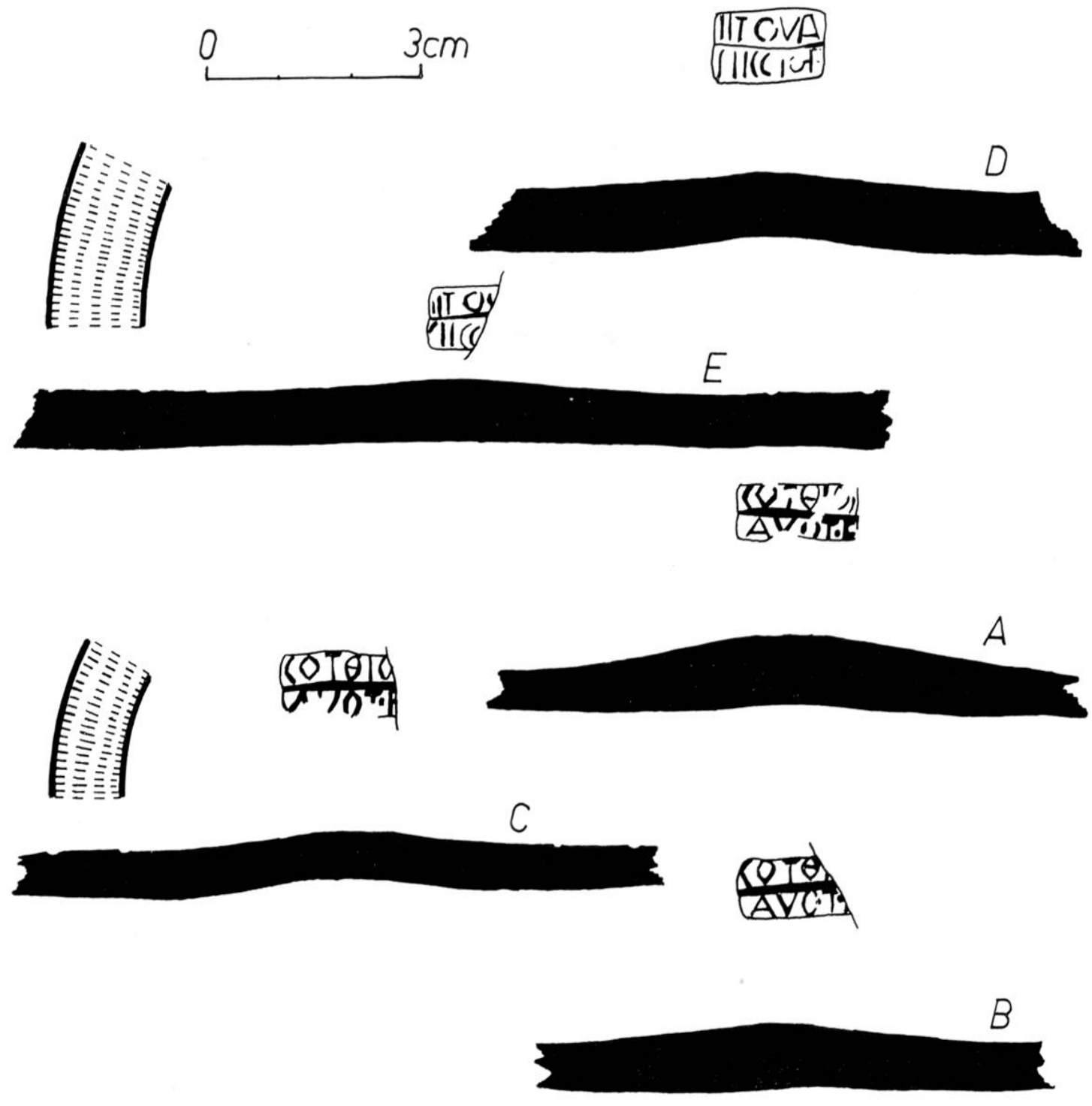

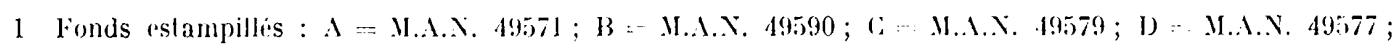
I: - I. ..... 49580.

$56 \mathrm{~mm}$ ); pâte gris clair ; surface interieure noirâtre. traces apparentes de lissage epargnant au centre une dépression circulaire (fig. 3. 3) : surface extérieure plus claire, lissage moins soigné, inexistant a l'intérieur du pied. (i : fragment d'un bol (?) (fig. 2, (i) marque (i) ? ISO (M.A.X. 49583) ; pied non rapporte (o extérieur : $67 \mathrm{~mm}$ ); fond epaissi vers le centre pate gris clair ; surface intérieure gris sombre. traces visibles de lissage; surface extérieure plus claire et moins soignée,

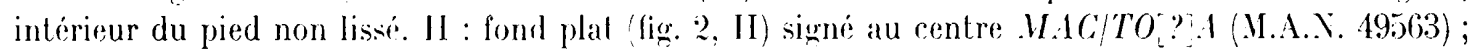
pied bas, faiblement dégagé. lris excentrique ( $\#$ extérieur : $101 \mathrm{~mm}$ ), allache arrondie d'un bord sans doule à peine oblique ; pate gris clair ; surface intérieure noirâtre, bien lissée ; surface extérieure

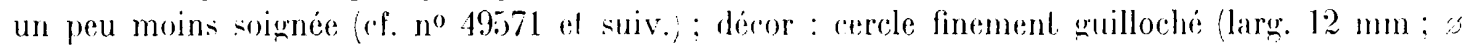
intérieur : $29 \mathrm{~mm}$ ) el deux cerces en relief $(\mathrm{s} 86$ et $90 \mathrm{~mm})$. I : fragment d'une écuelle (M.A.X. 19548) (fig. (7, lj; paroi oblique (epaiss. environ $7 \mathrm{~mm}$ ) à live tres saillante, légirement tombante et creusée d'une dépression sur sa face supérieure ; pied à peine dégagé ; fond plat détruit au centre ; 


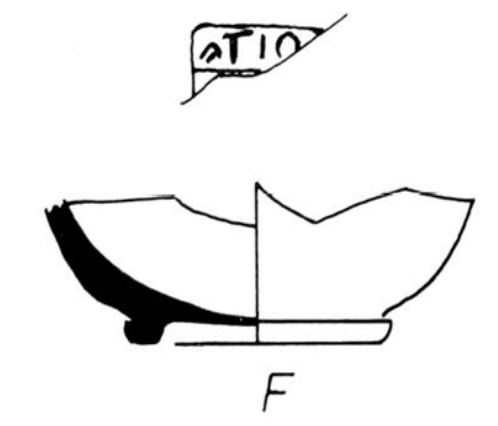

(SUKA10)
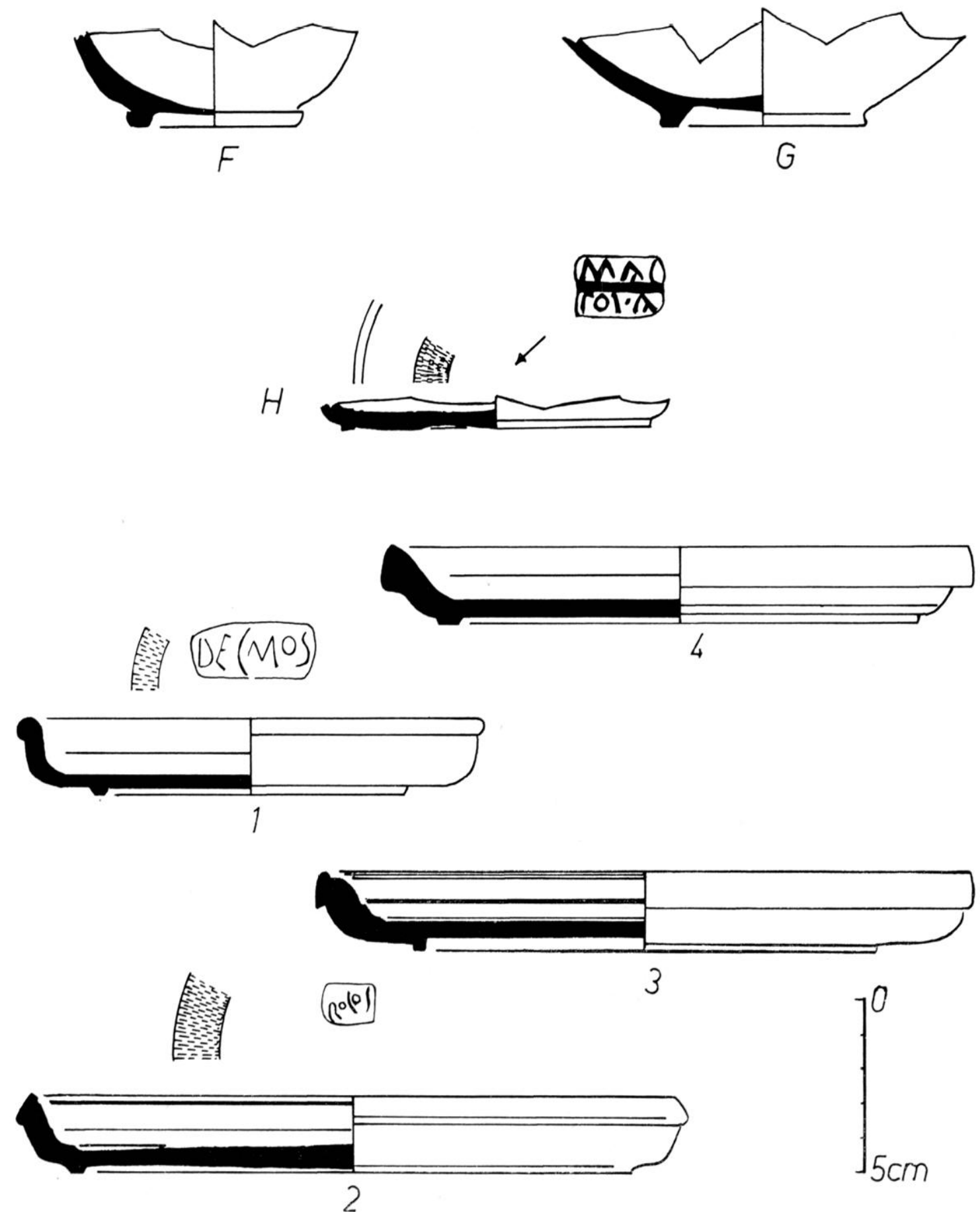

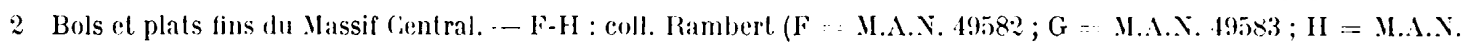
49563. Estampilles : éch. $1: 1,-1$ : assiette de Gergovie dapris II. LAbrocsse, op cil., 1. 12, fig. :23; ; : assiette

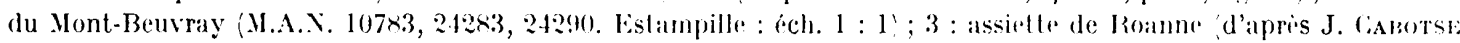

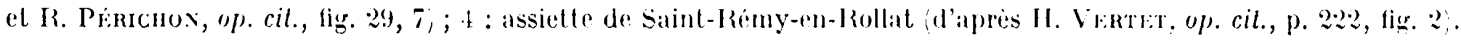


ø intérieur au niveau de la lèvre : $219 \mathrm{~mm}$, $\varnothing$ intérieur du pied : $138 \mathrm{~mm}$, haut. totale : $35 \mathrm{~mm}$; pâte el aspect intérieur : cf. $n^{0} 495633$; surface extérieure moins soignée, traces de lissage imparfait sous le fond ; à l'extérieur, sur la paroi et le fond : restes d'un gralfite (fig. 5) comportant au moins six lignes. Les cassures, comme les surfaces lissées de toutes ces pièces, sont pailletées de mica. Cet eflet est particulièrement sensible à l'intérieur des deux derniers vases.

La qualité du travail, l'aspect, la couleur de cette céramique la rattachent à une production fine, fumigée, courante en Gaule en particulier à partir de la Tène tardive*. Le décor de trois des fonds plats ${ }^{5}$ et l'estampille de huit des échantillons trahissent, en outre, l'influence des produits italiques sigillés ${ }^{6}$. Enfin, le libellé de plusieurs des marques? atteste une origine gauloise. Tous ces caractères incitent à rapprocher notre série de certains types de la céramique dite gallo-belge ${ }^{8}$. Cette dénomination d'une vaisselle répandue surtout dans le Nord et l'Est de la Gaule et en Angleterre ${ }^{9}$ n'est pas absolument satisfaisante, dans la mesure où elle rend compte d'un phénomène historique : la production d'une céramique fine et soignée dans des ateliers indigènes, au début de la colonisation, sur une aire géographique déterminée, beaucoup plus qu'elle ne caractérise étroitement un genre de fabrication. En effet, la poterie gallo-belge inclut, du point de vue de la typologie, des formes multiples, déterminées par des influences diverses -- la plus remarquée étant celle de la sigillée - . Et la même variété apparaît dans les techniques, puisqu'on rencontre, dans les mèmes services, des pièces engobées ou seulement lissées et de couleurs différentes - - du noir au rouge $\mathrm{e}^{10}$ - selon la qualité de l'argile et la nature de la cuisson. Cette double ambiguïté est en partic levée par ceux qui se fondent sur des critères typologiques et parlent, dans le cas d'une influence précise de formes déterminées, d'i imitations de sigillée "11, que l'on sépare, alors, des poteries fines contemporaines ressortissant à des traditions différentes. Certains, également, opèrent des distinctions techniques entre lerra nigra et terra rubra ${ }^{2}$. Il demeure que, en pratique, les bornes de l'imitation et de la tradition

4 Cf. en particulier J. B. WARD PERkiss, The pottery of Gergovia in relation to that of other siles in central and southwestern France, dans The Archaeological Journal, XCVII, 19.10, p. 37-87; J.-J. IATr, Essai d'une comparaison entre la céramique cellique d'Aulnal-Sud el la céramique gallo-romaine précoce de Gergovie, dans Bulletin hislorique et scienlifique de l'Auvergne, 1945, p. 151-174; du même, Aperçus sur l'évolulion de la céramique commune gallo-romaine principalement dans le Nord-Est de la Gaule, dans Rev. éludes anciennes, LI, 1949, p. 100-128.

5 G, E, H. Cf. par exemple, M. LABrousse, Les fonilles de Gergovie, dans Gallia, VIII, 1950, p. 45.

6 A-H.

7 Celles qui comportent, sous une forme plus ou moins abrégée, auot.

8 La définition, trop limitative, de .11. Labrousse, op. cil., p. \$2, doit être complétée par la référence aux études, entre autres, d'E. Ritterling (Das frührömische Lager bei Hofheim in Taunus, Annalen des Vereins für Nassauische Altertumskunde, $X X X, 1912$, p. 326-328;, J. Holwerda (I)e belgische Waar in Nijmegen, Ximègue, 1941, p. 117-120), W. Drack (Die IIelvetische Terra-Sigillala-Imilalion, Bâle, 19.45, p. 23-30.

9 Outre les travaux déjà cités, on compte parmi les publications essentielles : S. LolscuCkE, Keramische Funde in Haltern, Mitleilungen der Altertumshommission für Westfalen, V, 1909, p. 101 et s. ; T. MAY, Calalogue of the Roman Poltery in the Colchester and Essex .Musenm, Cambridge, 1931, p. 9 et s. ; C. F. C. Hawkes et .I. R. Hul., Camulodunum, Oxford, 1947, p. 202 et s.; H. Koютнe, Zur geslempellen belgischen Keramik aus Trier, dans Festschrift für A. Oxé, Darmstadt, 1938, p. s9 et s. (inventaire d'estampilles;.

10 Cif. par exemple, E. Rit'terling, op. cit., p. 333 , remarques sur le type $97 \AA$.

11 W. Inack, op. cit., et E. Éț.ingi:k (Die Keramik der Augsler Thermen, Ausgrabung 1937-38, Bàle, 1949, p. 42 et s.), entre autres, adoptent ce parti.

12 Voir pour ces définitions : Terra nigra: J.-J. Harr, Aperçus..., p. 110 ; Terra nigra et. Terra rubra: S. J. DE: LAET et H. ThoEx, Éludes sur la céramique de la nécropole gallo-romaine de Blicquy (IIainaul), III. La céramique belge à pále gris clair, dans Helinium, VIII, 1968 (1), p. 4. 
locale sont, parfois, difficiles à discerner et que la communauté des divers modes de fabrication ne rend pas la distinction plus aisée.

Le cas qui nous occupe pose le problème du développement, dans le Centre de la Gaule, de céramiques imitant la sigillée, alors que, jusqu'à présent, les exemples caractérisés et signalés comme tels paraissent assez rares. L'existence de notre série invite, d'autre part, à s'interroger, au nom de ressemblances techniques, sur les rapports qui unissent la vaisselle d'imitation et l'écuelle I.

Cé sont tantôt l'estampille, tantôt l'estampille et la forme, tantôt la forme seule, qui peuvent témoigner de la façon la moins discutable de l'influence de la sigillée sur certaines céramiques fines du Centre. Lne monographie a retenu, comme trace de cette production, une assiette noire de Gergovie (fig. 2, 1) marquée $D E C$ (i)MOS et qui s'apparente, ainsi que l'a montré M. Labrousse, au type gallo-belge 72 de Haltern et à la forme italique Ha 1 $(=\text { Goud. } 17 \text { et } 23)^{13}$. Un tesson informe, de qualité comparable, portant l'estampille $M A X(i) M O S^{14}$ provient, lui aussi, de Gergovie. On reconnaît à ces pièces une origine locale. On a signalé, d'autre part, à Roanne, plusieurs assiettes noires (fig. 2, 3), plus finement moulurées que celle de Gergovie, mais apparemment anépigraphes, qui relèvent de la même typologie ${ }^{15}$. Enfin, il ne faut pas oublier la vaisselle noire ou grise estampillée du Mont-Beuvray, que l'on connaît par les publications de J. G. Bulliot et G. de Mortillet, et dont j'ai vérifié plusieurs exemplaires au M.A.N. de Saint-Germain ${ }^{16}$. Par ailleurs une série nouvelle, celle des céramiques grises à bandes sombres, révèle le même genre de contamination. Je pense, du moins, l'avoir montré pour une assiette d'Angers, depuis longtemps publiée et considérée par J. B. Ward Perkins comme un exemple de la céramique à bandes lustrées - connue dans le Centre et le Sud-Ouest de la France -, mais très proche, par sa forme, du type signalé à Gergovie. Et il en va de même pour les deux tessons yris, ornés de zones foncées et claires el, en même temps, estampillés, qui proviennent de la collection Rambert ${ }^{17}$. Par conséquent, s'il est vrai que nos vases gris signés peuvent constituer un appoint notable pour ce petit groupe de Gaule du centre, ils ne seraient pas les seuls témoins d'une espèce, dans cette région. Aussi leur origine présumée n'a-t-elle pas lieu, a priori, de surprendre.

Comment doit-on les classer? Les cinq premiers fragments proviennent de formes

13 Op. cil., p. 42.

14 J. B. Ward Perkins, op. cit., p. 78-79, $\mathrm{n}^{\circ}$ 5. Peut-être faut-il altribuer au même potier le vase noir signé AVOT[---J/.MAXWV (CIL XIII, 10010, 249$)$ et trouve à Jublains.

15) J. CABotse, Essai de classification de la céramique "commune "gallo-romaine du sile de la "nouvelle poste" à Roanne (Loire), dans Cellicum, III, 1961 1962, pl. 37 (fig. 3, 1) et p. 120 ; J. Cianotse ot R. Périchon, Céramiques gauloises el gallo-romaines de Roanne (Loire), dans (iallia, XXIV, 1966, p. 65-67, fig. $29,7$.

16 J. G. Bulliot signale des vases d'OLICIOSS CIL XIII, 10010, 1458, DITAVIDITAV (CIL XIII, 10010, 786;, ATVCCVS Le Forum de Bibracte, dans Revue des Soc. Sav., 1877, p. 345-346; et publie des fac-similés de COTOKCOTOS?, IVOH, SEN?, [.jORIOS, AVRO, ROCOS' Fonilles du Mont-Beuvray. Ancienne Bibracte. Album exécuté sous la direction de $F$. et $N$. Thiollier, Saint-Étienne, l899, pl. L.IX), d'après les exemplaires du musée Rodin d'Autun. A Saint-Germain sont conservís des fragments envoyés par J. G. Bulliot et recensés par G. de Mortillet Inventaire des sigles figulins du musée de Saint-Germain-en-Laye, Paris, 1879, p. 18.. Ils portent les signatures IOV/IIRI ] ] M.A.N. 10784, RIOCOS (M.A.N. 10786), [.?OICOS (M.A.N. 10785), ROCOS (M.A.N. 10783) (second exemplaire ?. Ces marques ne sont pas signaliens dans les collections de Belgique, Allemagne, Suisse, Angleterre.

17 Op. cit., fig. 1. L'un des vase's est sans doute une jatte; l'autre tesson, mal conservé, pourrait appartenir à une forme analogue. 


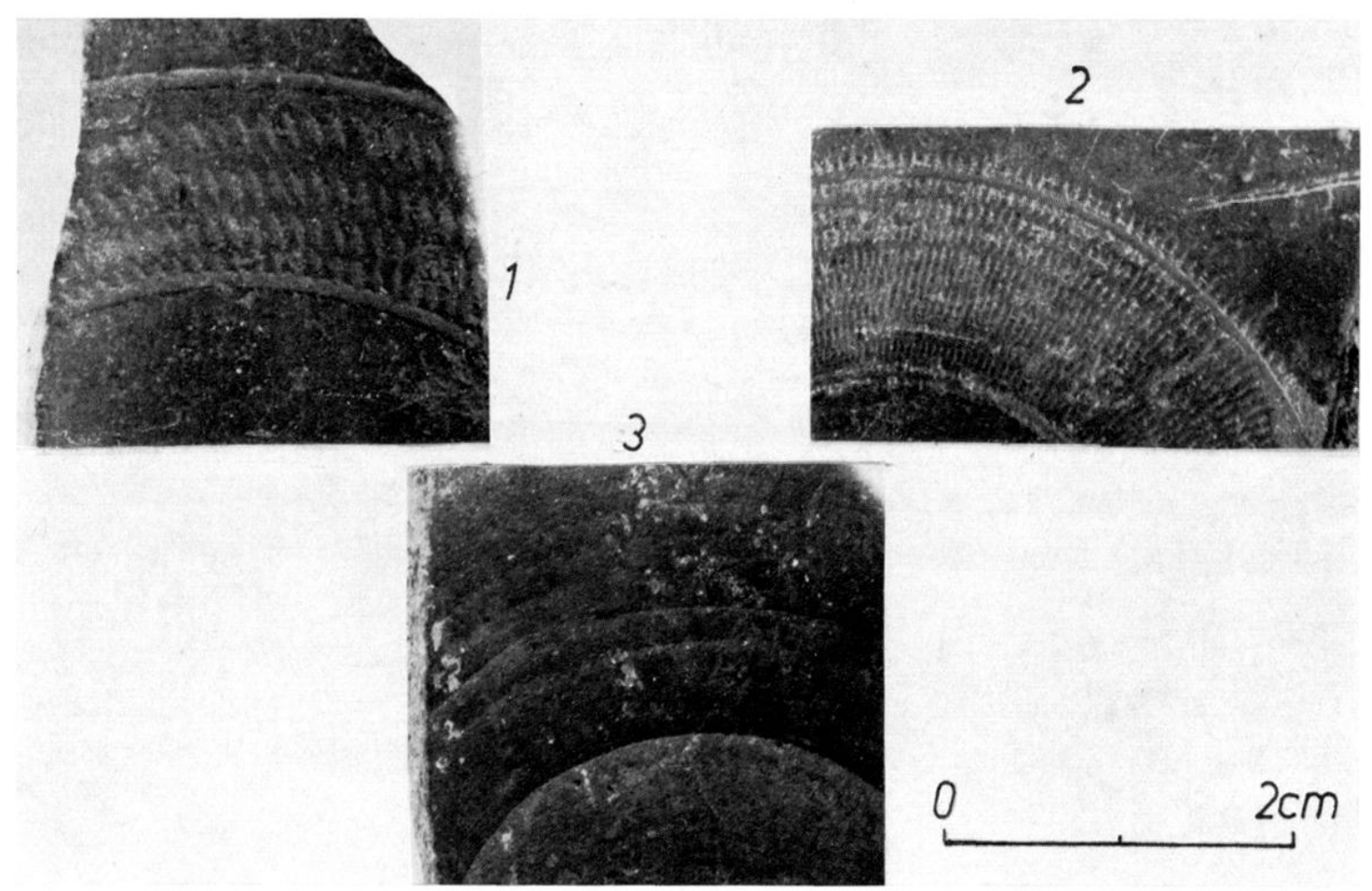

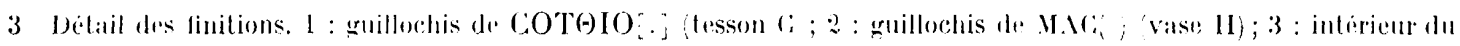
bol d'ATI-... vase $\mathrm{F}$ a la linite du lissage.

larres et robustes, si l'on considère à la fois l'épaisseur de la paroi et le diamètre - inférieur a l'original complet - des tessons conserves. Deux de ces fonds appartiennent a des assiettes ou des plats, à en juger d'apres le décor guilloché (fig. 3, 1 et 2), apparemment propre à ce lype de récipient. Les trois autres peuvent, eux aussi, relever de formes analogues, mais la faible surface qui subsiste, l'absence de fait de tout décor incitent à ardmettre concurremment l'hypothèse prudente de larges jattes. La qualité de la finition du dessous des trois fragments est en tous points identique, il est vai, a celle des formes plates avérées du groupe: outre les deux tessons ornés, les vases mieux conservés II et l. Et elle diffère, en revanche, de ce qu'on observe sous les pieds, plus etroits, des modeles creux. Toutefois, il reste a montrer que le lissage incomplet est une pratique liée à la forme même de l'assiette (au moins dans certains ateliers), plutôt qu'au diamètre de la surface à lisser, c'est-à-dire aux dimensions du vase, quel qu'en soit le type. Les autres tessons présentent des profils mieux conservés, sinon toujours plus aises à identifier. L'un (F) appartient à un petit bol, l'autre (G), à une jatte ou à un bol de plus vastes proportions (figr. 22, F-(i). Ils semblent être, pour le moment, parmi les fragments gris ou noirs imitant la sigillée, les deux seuls exemples de formes différentes de l'assiette signalés dans cette région ${ }^{18}$. Aussi n'est-il pas possible, compte tenu de ce qui reste de chaque vase, de proposer des restitutions ${ }^{19}$. Quant

18 .J jume preferable de considerer les pieces ornés comme ajpartenant a une espece un peu differente et dont les rapports avec la ceramique d'imitation classique sont a preciser, en particulier du point de vue de la typologia.

19 Nilleurs, Ies formes creuses, lisses, fre plus frefuemmont imities a hatute eporfue sont surtout les D. 21/25): 27 el 10 , tandis que sulus les lalaviens l'influence des b. 3x est particulierement sensible. 
au troisième (fig. 2, I), son fond plat, orné d'un cercle guilloché très finement, en fait, normalement, une petite assiette. Et le pied à peine dégagé, sous l'attache arrondie d'une paroi sans doute presque verticale, l'apparente plus étroitement qu'à tout autre à un type bien connu : celui des assiettes fines de Saint-Rémy-en-Rollat (fig. 2, 4), illustré également par l'assiette de ROCOS (fig. 2,2 ) trouvée au Mont-Beuvray ${ }^{20}$. Nous n'avons pas affaire dans tous les cas au même mode de fabrication : les échantillons de Saint-Rémy, d'après la description, paraissent n'être pas fumigés; le tesson de la collection Rambert a été cuit, comme l'exemplaire du Mont-Beuray, en atmosphère réductrice. Mais la ressemblance de forme n'est pas douteuse, entre tous ces vases, et n'a pas lieu d'étonner : j'ai souligné déja $a^{21}$ les similitudes générales, dans les proportions, les attaches, la mouluration du bord, entre la série de Saint-Rémy et les assiettes (une noire et une grise) de Gergovie et d'Angers. Une telle parenté permet, en tout cas, non seulement d'ajouter du crédil à la tradition relative à l'origine de la collection Rambert, mais d'imaginer, avec vraisemblance, ce que pouvait être, intact, le vase II. En ce qui concerne la datation, la typologie, comme il était prévisible, n'offre guère de ressources. Ln des analogues les plus proches, par sa qualité, de l'assiette Rambert : l'exemplaire de Gergovie, est placé à l'époque augustéenne ${ }^{22}$. Et il doit en aller de même, du moins selon l'opinion la plus générale, pour l'échantillon du Mont-Beuvray ${ }^{23}$. Pourtant, s'il est permis de présumer de la ressemblance de ces pièces avec II, il n'est pas possible de déterminer dans quel rapport chronologique se trouvent les trois vases : les informations manquent sur ce type et son évolution, et II est très détérioré. Un point paraît, toutefois, assuré quant à la durée probable d'une telle production : cette forme d'assiette, attestée en cérarnique fine fumigée, est datée de la première moitié du I $^{\text {er }}$ s. à Roanne, tandis que les vases, non fumigés, de Saint-Rémy-en-Rollat sont situés à la fin du règne de Tibère ou au début de celui de Claurle ${ }^{24}$.

Les autres fragments plats sont trop incomplets pour qu'on en puisse rien tirer de sùr. Les observations d'E. Ritterling sur les guillochages du matériel de Hofheim pourraient inciter à placer $C$ et $\mathrm{E}$, aussi bien que $\mathrm{II}$, dans le premier tiers du $\mathrm{I}^{\mathrm{er}} \mathrm{s}^{25}$. Toutefois, ces constatations, valables sans doute pour la région où elles ont été faites, n'ont encore qu'un raractère local et devraient être ailleurs largement confirmées avant d'être utilisables comme critère général de datation. Aussi ne me parait-il pas possible de me fonder sur cet indice pour fixer aux échantillons du Centre un lerminus ante quem. Quant aux deux formes creuses, je ne leur connais actuellement aucun analogue publié et daté dans la

20 II. Vertet, Céramique commune de lofficine de Suinl-Rémy-en-Rollul, dans Gallia, XIX, 1961, p. 222, fig. $2 ;$ p. 221 et 223. L'assiette du VIont-Beuvray ne figure pas dans l'album des fouilles. Je l'ai reconstituée à l'aide de trois tessons qui se recollent parfaitement ent ro cux, mais se sont trouvés dispersés, apparemment, des les expéditions de Bulliot all musée.

21 Op. cit., p. $74-76$.

22 M. Labrousse, op. cil., p. 19.

23 Cf. par exemple, E. ErTuNian, Vorbemerkungen zu einer Diskussion des Aleius-Problems, dans Rei Cretariae Iomanae Fautorum Acta IV, 1962, p. 35 at 37, n. 20.

24 J. CABotsl, op. cil., p. 117 ; H. VнRTLT, op. cil., p. 2:25.

25) Voir, à ce propos, le résumé des remarques d'E. Kittring sur la céramique d'I Iofheim que puhlie J. Fromols (Fragments de céramique romaine (époque fauienne) Irouvés au jardin du Luxembourg, dans Rev. archéologique, 1959) $(\mathrm{II}$, p. 80$)$. 


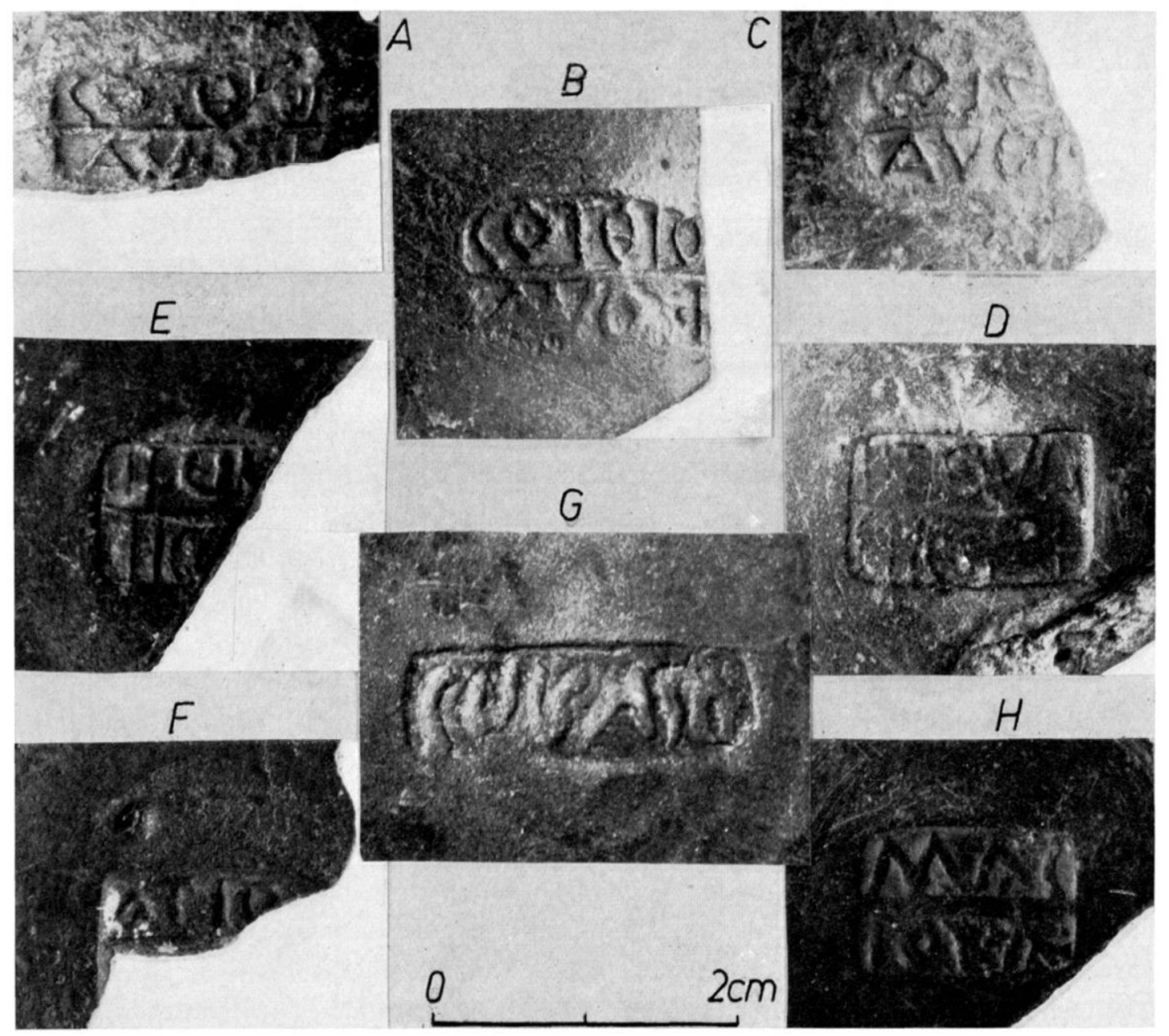

4 Agrandissernent des estampilles de la coll. Rambert. $\Lambda, \mathrm{B}, \mathrm{G}:$ poincon de COT $\Theta \mathrm{IO}[$.$] ; I), E : poincon dt:$ SECCIO ?[---] ; : : poincon d'ATI[---] ; $\mathrm{G}$ : poincon de CO[?]ASO ; H : poinçon de MAC: $)$.

zone de leur licu de découverte présumé. Ce sont donc les estampilles qui doivent offrir, dans cette enquête, les plus grandes ressources.

Inégalement conservées, plus ou moins bien dessinées ou imprimées, elles posent, d'abord, pour la plupart, des problèmes de lecture.

La premiere (fig. $1, \lambda-\mathrm{C} ; 4, \lambda-\mathrm{C})$, qui figure sur trois fragments $(A, B, \mathrm{C})$, est diversement brisée. Elle comporte deux lignes :

1. 1. $\Lambda: \operatorname{CO}[.] \Theta[] O.[]:. \mathrm{B}: \operatorname{COT} \Theta[-\ldots ; \mathrm{C}: \cot \Theta I O[-\ldots]$

1. 2. A : AVOT.[.]; B : AVOT.[--; $\mathrm{G}:$ IVOT. $[--]$.

La comparaison des trois exemplaires donne la longueur de chaque ligne et permet de proposer pour chaque échantillon une restitution plus précise. La ligne 1 comprend sept lettres : COTOIOL.: n'est pas douteux; le fragment de la dernière lettre, conservé par A, peut appartenir à un $I$ ou un $S$ un peu écrasé. La seconde ligne est faite de six signes : quatre lettres, un point, une lettre. $A V O T$. est indubitable. Pour la derniere lettre, la comparaison de A, qui conserve le haut d'une haste et deux barres horizontales, et de $\mathrm{C}$, où l'on voit la haste dans son entier et l'amorce des deux mèmes 
harres, autorise à exclure $I^{\prime}, R, E$ et a ne retenir que $F$. La forme la plus complète de la signature est done COTAIO [i ou sil AlOT.F.

La deuxieme estampille (fig. 1. D-E; 4, I)-E), conservée sur deux tessons, comporte igalement deux lignes:

l. 1. Г) : IITOYA: L: IITOIF- - :

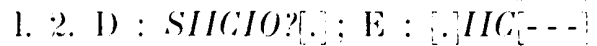

Ia premiere ligne est entierement conservée par I). Compte tenu de l'ordre indifférent observé dans ces marques, elle peul et doit, vraismblablement, être lue, de droite à qauche, AVOTE. forme bien attestée et relatirement intelligible, ce qui n'est pas le cas de ETOV $A^{26}$. La comparaison des deux exemplaires, l'un amputé, l'autre à peine imprimé. permet de rétablir à gauche de la seconde lignne SIICI. Le tesson D, très flou, conserve apparemment un $O$ apres l'I. On distingue ensuite une haste, peut-itre barrée au sommet et rayée. semble-t-il. accidentellement, vers son milieu. d'une trare horizontale. On peut done proposer IITOVAISIICIO? "est-i-dire AVOTE/ SEC:Cilo? ?

La troisieme estampille (fig. 2. F ; 4, F) avait deux lignes. Il reste, sur le tesson F, une partie de la première. L'amorce de l'angrle supérieur droit du cartouche, seule conservée, ne paraît laisser la place que pour une lettre, deux a la rigueur (si toutefois cet espace a jamais été occupé), au-deli

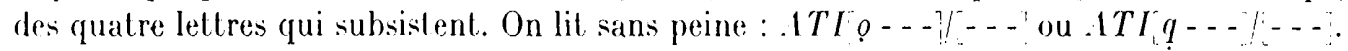

La quatrieme estampille (fig. 2. ( $;$; 4, (a), complete sur le fragment $G$, est mal tracée. Elle ne comprend qu'une ligne où l'on discerne. sans trop de mal. CO.ASO. I.e troisieme signe, plutòt qu'un $Y$, très improbable, pourrait être un $C$. précédé soit d'un tracé accidentel, soit d'un autre $C$, déformé. K. Marichal, à qui j'ai soumis ces inscriptions, ne peut proposer comme certaine aucune de ces interprétations. On supposera done $C O\left[., 4 S O\right.$ ou $C O^{\circ}$. . ASO.

La dernière tstampille (fig. $2, \mathrm{H} ; 4, \mathrm{H}$ ), inscrite sur deux lignes, est également douteuse :

I. 1. $M A C$

l. 2. $T O^{-} .4$ ou $T O[]$.

Entre l'o et l'A on distingue une oblique légerrement courbée, en haul, vers la gauche et une sorle de gros point pris de l'A. La surface tres accidentée, à cel endroit permet de croire à une détérioration de l'estampille ou de la matrice. Deux hypotheses s'offrent à nous : la 1. 2 est réellement

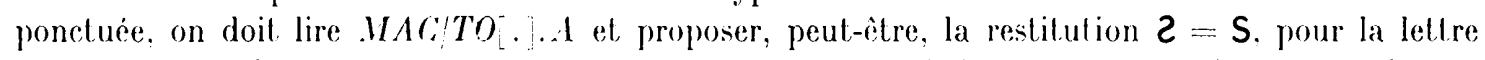
qui suit l'o: la 1.2 non ponctuée, porte en fait entre $O$ et $A$ deux fragments d'une mème lettre. Pour qui considere la forme du plus étendu, il peut s'agir d'un $V$. Nous aurions alors. $M A C / T O[u] A$, c'est-i-dire, selon loute vraisemblance. $M A C() / A V O T$ (pour les raisons qui nous incitaient à lire, plus haul, IITOVA: AVOTE). Le mauvais état de l'estampille conservée par le seul tesson II et la difficulté i interpréter la haste oblique, si on l'isole, font pencher pour la seconde solution, sous réserve d'aulres découvertes ${ }^{27}$. De toutes façons, le contenu de l'inscription ne change pas triss sensiblement, puisque, dans les deux éventualités, il comporte, l'un ou l'autre abrégé, un nom propre et le mot auol. si les formes les plus courantes de ce verbe ou substantif sont auol ou auot ${ }^{28}$,

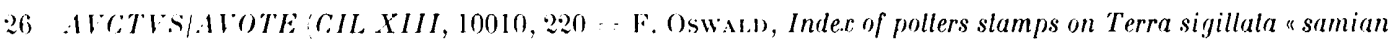
mare ". Margidunum, 1931, p. 31 ; ALCISS/AVOTII, rectification de la lecture fautive précédemment publice :

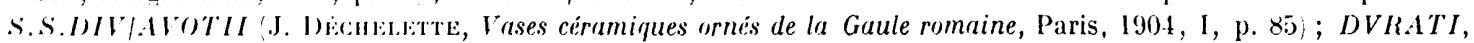

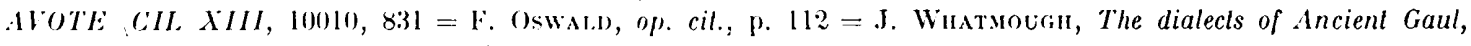
Ann Arbor, 1951, p. 341, provenant de Lazoux.

27 Ine eslampille, signalé all $C I L . X I I I, 10010,2871$, presente qu'lque ressemblance avec la nòtre et parait provenir de la même région Varennes Moulins apud Esmonnot,. Elle est malheureusement encore plus deteriorese

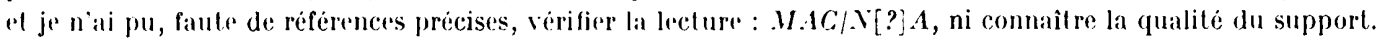

2o J. Whatnoegill, Gaulish TLOGOS, ACOT, IELRL, dans Journal of Cellic Sludies, I, 1950, p. 10. 
on rencontre aussi auo, $a u^{29}$ et $a$ n'est pas inconnu ${ }^{30}$. En effet, le développement en auol' ) fournit apparemment la seule justification de cette initiale isolée, en Gaule, sur de la céramique signée non " arétine».

Les trois signatures dont les deux lignes subsistent sur nos tessons peuvent donc, avec plus ou moins de vraisemblance, ou de certitude, contenir la formule auol(), considérée comme gauloise et attestée sous des formes plus ou moins développées. La question n'est pas tranchée de savoir s'il s'agit d'un verbe ou d'un substantif, ou tantôt de l'un, tantôt de l'autre ${ }^{31}$. Les exemples jusqu'ici signalés montrent que ce mot est employé, pour signer, par des artisans - potiers ou autres - là où d'ordinaire on rencontre le plus souvent fecit. On trouve, d'ailleurs, l'un ou l'autre mot employés alternativement par le même signataire ${ }^{32}$. Dans le cas présent, la moins douteuse des marques, la première, paraît introduire une nouveauté par le fait qu'auol et $f$ figurent ensemble sur la même estampille. Il est très improbable, compte tenu de la rareté et de l'ancienneté de l'emploi de figulus apposé, dans les signatures italiques, et aussi de la longueur habituelle des abréviations, qu'il faille développer $f$ autrement qu'en $f(e c i l)$. La question est donc de savoir si l'on a ainsi une preuve qu'auol() peut être un substantif - sujet de fecit - ou s'il s'agit plutôt de doublets employés concurremment et imputables au bilinguisme. Ce serait, à ma connaissance le premier exemple de ce genre. Nais il est évident qu'une forme moins compatible qu'auol avec une flexion verbale permettrait de plaider plus aisément en faveur de la première des hypothèses.

La forme prise par les poinçons appelle, aussi, quelques commentaires. En effet, les estampilles inscrites sur deux lignes sont généralement considérées comme anciennes et l'on s'entend, d'ordinaire, pour penser que ce type de marque, usité en Gaule surtout à partir du début de notre ère, ne se rencontre guère après les dernières années du règne de Tibère : c'est, du moins, ce que suggèrent, dans leur ensemble, les signalures sur sigillée et sur céramique d'imitation jusqu'à présent publiées et datées. Donc, si l'on se fonde sur ce critère, sept des huit tessons marqués peuvent provenir de vases fabriqués entre la dernière partie du règne d'Auguste et environ 30/35. Le dernier est plus difficile à dater par le même moyen, car il existe de nombreux poinçons sur une ligne, contemporains des précédents, en Suisse ou en Allemagne, et on en trouve également à Gergovic et au MontBeuvray. Le nom, bien que tracé ici en lettres inélégantes, est à peu près lisible et diffère des séries de jambages dénuées de signification, qu'on voit apparaître dès le règne de

Cf. AVO: Asialicus (Lezoux) : Oswald, op. cil., p. 24,

Cassalus (Est) : $\quad-\quad \quad-$, p. 64,

Cilos (Est) : J. Whatmovgin, Dialects..., XXXIX, 677,

[---]orti (Est) :

...

AV : Rulenus (Lezoux) : Oswalb, .., p. 271 ... CIL XIII, 10010, 1670,

Inticilu (Lavoye) : J. WhatmovaH, ibid. $=$ CIL XIII, 10010, 1035,

Ericu : CIL XIII, 10010,859,

pour n'envisager que les signatures de potiers.

30 Lucius a: W. Drack, op. cil., pl. XVII, 55,

Lullo a : J. Whatmough, op. cit., XLIX, 872,

Togos a : CIL XIII, 10010, 1926 (Lezoux).

31 J. Whatmolgh, Gaulish..., p. 10.

32 Rutenus fecit, Ruten. fe et Ruten au, attestés à l.ezoux (Oswald, op. cil., p. 271). 
Claude sur des sites connus ${ }^{33}$. L'estampille, assez grande, est enfermée dans un rectangle allongé, aux extrémités convexes. Malheureusement l'absence de termes de comparaison locaux en assez grand nombre et le fait qu'ailleurs on constate l'existence de particularismes régionaux, au niveau de ce genre de caractéristiques secondaires ${ }^{34}$, ne permettent pas de savoir quelle portée on peut donner à de telles observations : en Suisse, par exemple, elles s'appliquent à des prorluits d'époque flavienne, mais n'ont pas nécessairement, en d'autres contrées, la même signification. Il n'est donc possible que de constater que quatre des estampilles sont, sans nul doute, anciennes, tandis que la cinquième présente une forme générale banale, en usage, sur ce type de vaisselle, d'Auguste aux Flaviens, et des particularités qui, en certaines parties de la Gaule, la feraient considérer comme relativement tardive.

Ces signatures, enfin, livrent le nom de quelques potiers et l'on est en droit de se demander quels rapports ceux-ci entretiennent avec des ateliers déjà connus. Il faut noter d'abord, qu'aucune des marques ne figure dans les grands recueils de fac-similés gallo-belges que j'ai eus entre les mains. lit les relations entre nos estampilles et les fabriques de sigillée ailleurs connues en Gaule demeurent imprécises. Il n'est que de passer les noms en revue pour le montrer. ATIO seul ou en composition n'est pas attesté dans les ateliers du Centre ou du Sud de la Giaule ${ }^{35}$. ATTIVS est connu, mais rien ne permet de conclure à des rapports autres que possibles, avec notre artisan, compte tenu de l'imprécision de la datation et du caractère incomplet de notre marque ${ }^{36}$. Un COCASVS est signalée ${ }^{37}$, mais on ignore son origine et le moment de son activité, et, surtout, la lecture de l'estampille Rambert n'est que conjecturale. COSIVS a travaillé à la Graufesenque ${ }^{38}$. Toutefois, la fréquence des noms dérivés d'un *koutso, que permettent de restituer à la fois la graphie maladroite de notre marque et la série de noms comme COSIVS, COSSIVS, etc. ${ }^{39}$, autorise surtout à envisager l'existence possible de multiples homonymes. De plus la datation assez approximative de COSIVS, le potier en sigillée, interdit tout rapprochement vraiment fondé. Nombreux paraissent, parmi les artisans, ceux dont le nom commence par $M, A, C$, de MACCALVS de Lezoux à MACVS de la Graufesenque, en passant par MACIRVS de Vichy (?"40. Aucun MACTOS n'est attesté et seul MACCARVS de la Graufesenque pourrait avoir quelque rapport chronologique avec notre potier ${ }^{41}$. Enfin, on relève dans des inscriptions les mentions de SECCIO et SECCIV $S^{42}$, mais on ne trouve pas ces noms

33 Voir, par exemple, E. Ritteriswa, op. cil., p. 328-329, fig. 84.

34 W. Drack, op. cit., p. 41.

35) Voir, on revanche, CIL II, 5855; XIII, 4230, 10001 (353), 10017 (183; ; Alio est égałlement signalé (CIL III, 5101 .

36 La Graufesenque (Claude-Néron); I,ezoux (Trajan-Hadrien) = Oswal.o, op. cil., p. 394 et 29.

37 CIL. XIII, 10010, 594.

38 (oswat.d, op. cil., p. 89, 376-77, 126 (epoque flaviennc).

39 Ci. A. Holdir, Altceltischer Sprachschatz, Leipzig, I, 1896, cos- : 1138-1140; cus- : 1206-1208. Ce radical, selon I. Iejeune, peut être attesté également par un Coddocatlus authentique ?; a Besançon et Coddis à Langres (IOL.DER, op. cil., 1061).

40 Oswai.d, op. cit., p. 173-177.

41 Tibère-Néron (ibid., p. 173).

42 A. Holdir, op. cit., III, 1921, 1423-1424. 
parmi les marques ${ }^{43}$. Et, d'ailleurs, l'état des estampilles ne garantit pas la forme exacte du patronyme qui nous occupe. Ainsi l'examen du contenu des signatures conduit surtout à tenir ces dernières pour étrangères aux inventaires de sigillée d'imitation du Nord et de l'Est de la Gaule, sans que les marques paraissent davantage se retrouver en Angleterre. Pour négatif qu'il soit, un tel résultat a comme conséquence de n'infirmer en rien la tradition relative à l'origine de la coll. Rambert et même de confirmer la possibilité que ces vases aient été non seulement découverts à Vichy ou dans les environs, mais même produits dans des ateliers assez proches. L'absence de relations entre ces potiers et ceux des ateliers de sigillée les plus voisins ne saurait constituer une objection : de tels rapports ne sont qu'extrêmement rares et conjecturaux et l'on commence à peine à découvrir les débuts des fabriques gauloises de sigillée classique. Cependant l'hypothèse de l'origine locale de nos tessons repose essenticllement sur un argument ex silentio, dont la faiblesse est toujours à craindre, et aussi sur le fait, ailleurs éprouvé, que ce matériel n'avait d'ordinaire qu'une diffusion limitée ${ }^{44}$. En l'occurrence, il est possible toutefois que nous ayons, par une autre source, la trace d'un de nos potiers dans le Massif Central45. En effet, A. Holder signale ${ }^{46}$, comme "Terra-nigra Stempel aus der Auvergne ", COTOIO AVOTI. La ressemblance entre cette estampille et $C O T \Theta I O / \Lambda V O T \cdot F$ et le fait que le support soit de même nature dans les deux cas incitent à se demander si la différence entre les deux noms n'est pas due à une erreur de lecture - le $\Theta$ étant extrêmement voisin de l'O ou à un défaut dans l'impression de la marque, ou encore si une confusion, imputable a l'ignorance, n'a pas conduit le graveur de poinçons à transformer indûment un $O$ en $\Theta^{47}$ ou, inversement, un $\Theta$ en $O$. Selon V. Lejeune, a qui j'ai soumis ce problème, un lapsus COTOIO- pour * ${ }^{*} O \Theta I O$ serait pensable, un lapsus COT $\Theta I O$ - pour COTOIO-, peu vraisemblable. Il demeure, cependant, que COTOIO-, en soi, n'est pas impossible, bien qu'il ne soit pas attesté. Quant au second élément de la signature, il paraît trop différent de celui que nous lisons, pour que nous puissions penser qu'A. Holder n'a signalé qu'un nouvel exemplaire de la même marque, et rien, non plus, n'autorise à croire que la sourre de son information est le matériel Rambert. Mais il est regrettable qu'en la circonstance force soit de se fier à lui. En effet, lorsque j'ai voulu vérifier l'estampille, il m'a été impossible de savoir ce que signifiait la référence vague fournie par cet auteur : "bei Bohn ». Je n'ai pu que m'assurer qu'il ne s'agissait ni du catalogue de l'instrumentum du CIL XIII, ni de la longue préface consacrée par (). Bohn, dans ce volume, aux remarques sur les vases estampillés. Donc il n'est pas exclu que la transcription invérifiable COTOIO soit due à une erreur de lecture ou de graphie, voire à un accident, qui aurait défiguré un COT $\Theta I O$ philologiquement justifié ${ }^{48}$, et que, par conséquent, une signature de notre artisan soit,

43 In Secco(s) est signalé dans l'Est, entre Domitien ol Hadrien (CIL . YIII, 10010, 1756).

44 Voir, en particulier, les observations de W. Dnack, op. cil., p. 15-48 et 103-122.

45) Cif. en outre l'exemple possible noté précédemment. 'n. 27 !.

46 Op. cil., III, 870-871.

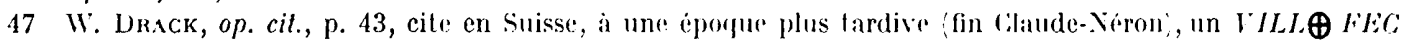
substitue a VILLO FEC, sous l'influence, malencontreuse mas probable, du $\Theta$, que l'on trouve dans des noms comme ML巴ILLYS.

48 la graphie -Te- ne semble pas actuellement avoir encore ete signaléc. Mais, selon M. Lejeune, elle est tout

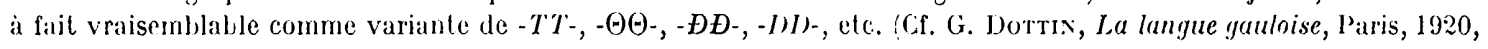
p. 62 ;. 


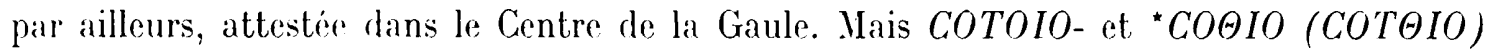
ont pu, aussi, coexister comme noms de deux potiers.

La dernière pièce de la série (fig. 6, I) se distingue des autres en ce qu'on ne peut l'associer directement à elles par sa typologic : cette écuelle est trop mutilée pour qu'on sache si elle comportait une estampille et elle relève d'une forme à laquelle on cherche rainement des modèles sigillés classiques. Il n'y a donc même pas de raison logique de supposer qu'elle ait pu ètre signée. L'intérèt qu'elle éveille d'abord tient au graffite qu'elle porte et qui semble n'aroir pas attiré l'attention. Cet indifférence paraît invraisemblable. Pourtant, quand on se reporte a l'ouvrage consacré par Mallat et Cornillon à l'Histoire des eaux minérales de Vichy ${ }^{49}$, on demeure indécis. On trouve bien, en effet, le récit de la découverte, par A. Rambert, vers 1880, d'une écuelle de même type, présentant une inscription à certains égards comparable ${ }^{50}$. Mais les différences de détail - - place el nature de l'inseription, nombre des lignes, situation respective des mots lus par Rambert et qu'on

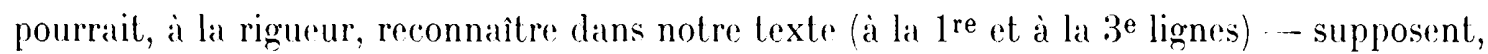
même si chacune s'explique, tant d'inexactiludes ou d'imprécisions dans les notes de Rambert, ou dans l'adaptation qui en est donnée, qu'on ne peut sans hésitations considérer qu'il s'agit d'un seul et même objet. Et supposer que l'assiette a subi des détériorations depuis sa mise au jour permet, peut-être, de rendre compte de la lecture ancienne, mais ne résoud pas, bien au contraire, les problèmes posés par la description du texte. Quoi qu'il en soit, l'état de l'inseription de Saint-Germain n'a pas permis encore de déterminer la signification de celle-ci ${ }^{51}$, ni, a forliori, la raison de sa présence sur le vase. Je me bornerai donc à proposer la transcription hypothétique que R. Marichal a bien voulu vérifier et compléter. Le graffite (fig. 5) comprend actuellement six lignes, peut-être la trace d'une septième : trois sur la face extéricure du bord, trois (ou quatre) sous le fond. La raideur du tracé, les retouches et les écaillages sur les bords des traits montrent que l'inscription doit avoir été faite après cuisson.

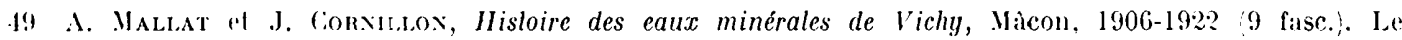
1)r M. Vauthey a eu l'extrême amabilite de me faire parvenir une copie du passage qui m'intéressait.

50 Op. cit., I, p. 34-35: "Il y a plus de vingt ans, Aymé Rambert, archeologue à Vichy, découvrit a l'extrémilé nord de la rue Iesbrest pres de la maison Vexenat. (1) dans un puits funeraire gallo-romain la moitic d'un plateau en terre noiratre, de vingt centimc̀tres de diamètre environ. Sa forme chail celle d'une assielte profonde el a bords plats. Sous le fond, se lisait une inscription tracée à la pointe avant la cuisson. Mais, le plateau n'étant pas entier, l'inscription, qui formait trois lignes circulaires, était forcément interrompue. Apres réflexion, Rambert parvint a déchiffrer et à compléter un nom propre qui lui était familier, cclui de Quilus, précédí de l'adjectif inclitus. A côté de ce plateau, il ramassa un petit bronze de dix à douze centimetres de haut, qu'il vendit en 11 ai 1895 an .Musio du louve et qui est devenu le Malade tenant un gobelel, malade fort connu maintenant de tous ceux qui s'oceupent d'archeologie medicale gallo-romaine. Rambert pensait que ce bronze representait Chilus, goutteux et souffrant, qu'on aurait modelé un vere a la main pour indiquer que, de son vivant, il se soignait en buvant des eaux minerales ; Chilus qui, un jour, avait ecrit sur une grande luile a rebord, decouverte dans le quartirr de Ballore : "Ego, Quilus, donali figulis amphoram vini "(2). - (1) Rue J)esbrest no 30. - (2, "Moi, fihilus, j'ai donne aux potiers une amphore de vin ", d'après Aỵme Rambert. Notes inédites.

A. Monty: op. cit. (reproduit dans Vichy gallo-romain, Macon, 1957, p. 235-236), signale: que la lecture de l'inseription sur brique est fautive - - ce que j'ai pu verifier - et met en doute le dechiffrement du graffile sur l'assiette. Mais il ne semble pas avoir eu la piece entre les mains, on du moins fonder sa critique sur une etude epigraphirgue du texte.

5l M. I.ejeune, qui a bien voulu l'examiner, n’a jugé possible d'en proposer aucune interprétation. 
162

GOLETTE BÉMONT
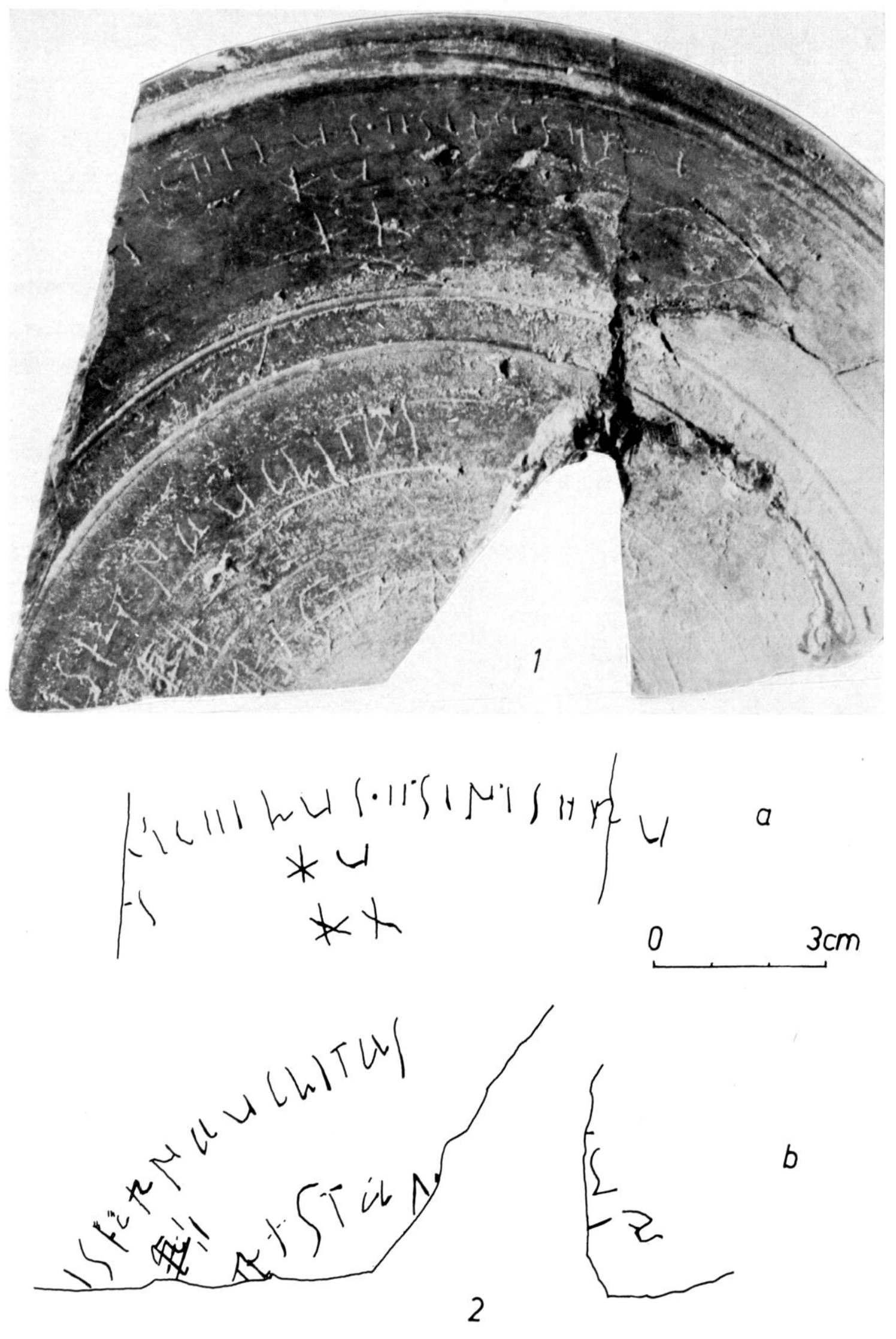

5 Graffite du vase I (M.A.X. 495.48) 1 : face extérieure du plat ; 2 : fac-similé (a : inscription du bord ; b : inscription du fond). 
l. 1. [- - I $\frac{I ? \text { CIELVS. ESINISERV }}{\operatorname{R} ? E I}$

10.325

1. 2. [- - - ]S deniers ;

I. $3 . \quad$ deniers 10

I. 4. [- - I?S. RNOVCLITOS

1. 5. $[--\overparen{E R I}$ ?

1. 6. [- - - RISTOON

I. $7 .[--]$ ?

I. I : la seule ponctuation assurée se trouve apries $-L I S$; 1 . 5: la premiere lettre conservée parait le résultat d'une surcharge, on peut distinguer un $E$ (non archaique) et un $R ; 1.6:$ sous le pied de la premiere lettre. on voit un trait vertical : un fragment de leltre?; au-deli d'une cassure apparaissent des signes, qui appartiennent aux 1 . j) el f 6 et sont demeurés indéchiffrables.

Les scules remarques qu'aient suggérées ces bribes de texte sont de deux ordres : -lus et -los pourraient être des terminaisons de substantifs ou d'adjectifs; le signe du denier suivi de chiffres ne peut guère impliquer qu'un compte ou un prix.

La qualité et la forme du vase offrent un peu plus de ressources. Cette pièce est une des plus soignées du groupe, avec l'assiette $H$ et elle présente aussi avec celte dernière des affinités particulières : aspect et nature de la pâte ${ }^{52}$, attention apportée au traitement de la surface ${ }^{53}$ permettent d'associer ces échantillons, alors que les sept autres fragments, pourtant voisins, diffèrent par une technique moins parfaite et trahissent même des irrégularités dans les petites séries marquées de poinçons identiques ${ }^{54}$. D'autre part, I est assez bien conservé pour qu'on restitue à peu près complètement son profil. Ciette variété d'écuelle à lèvre épaisse, horizontale ou légèrement tombante et souvent creusée d'une dépression sur sa face supéricure, est attestée actuellement sur plusieurs sites, en Gaule. On la signale, en particulier, dans le Jura, près de Dôle ${ }^{55}$ et dans le Nord-Est du Massif

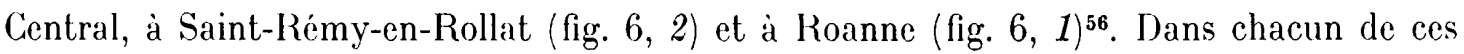
cas, les écuelles à pâte grise sont associées à des assiettes imitées de types sigillés classiques. A Lavans-lès-Dôle, il s'agit d'un matériel de dépotoir provenant d'un habitat gallo-romain daté de l'époque de Claude. La qualité apparente des écuelles et assiettes - "assiettes imitées des formes de sigillée, en terre grise ou gris-bleu $\|^{57}$ - amène à les classer ensemble

¿2 Ainsi la qualité de la pàte 'grossièrej, l'abondance du quartz el du mica grossiers, la nature de l'argile, le pourcentage du fer (env. $2 \%$ ) rendent ces tessons très semblables.

53 Les vases, couverts d'un faux engobe, ont été soigneusement lissés à durcté cuir. La couteur très sombre est due principalement au carbone.

54 Par exemple, la qualité du dégraissant (mica et quartz grossiers et abondants), le pourcentage de fer (env. $3 \%$ ) apparentent $\mathbf{C}$ à F, plus qu'à $A$ et B. D'autre part, D et E, signés du même poinçon, diffèrent par de nombreux traits, en particulier par la nature du degraissant mica et quartz asse fins et abondants dans un cas, absence pres(jua totale de mica dans l'autre) et le pourcentage du fer $(2 \%$ pour l'un, env. $4 \%$ pour l'autre $)$. B et G, en revanche, sont tris proches l'un de l'autre.

55 P. Petreporiv el J.-L. Onocze, Habilat romain à Moulin-Rouge, commune de Lavans-lès-Dôle (.Jura), dans Rev. archéol. Est, $X I X, 1968$, p. 262, 264, fig. 8, 11.

56 H. Vertet, op. cil., p. 219 et 223 ; p. 222 , fig. 3 ; J. Cabotse el R. P'́richox, op. cit., fig. 30,1 et p. 67.

57 Op. cit., p. 262. 
dans la même catégorie. A Roanne, les découvertes faites en divers points de la ville, en particulier sur le site de la nouvelle poste, ont permis de définir plusieurs variétés de céramique commune: les écuelles à lèvre et les assiettes d'imitation entrent, les unes et les autres, dans le mème groupe, celui de la céramique fine noire, caractérisée par un lissage soigné et un lustre très brillant ${ }^{58}$. A Saint-Rémy-en-Rollat, où il semble qu'on ait affaire à un centre de production, les écuelles sont grises, les assiettes rosées ou jaunàtres ${ }^{59}$. Du point de vue de la typologie, les écuelles les plus étroitement apparentées à celle de Saint-Germain sont celles du Massif Central, en particulier à cause de la mouluration du bord. Pour les assiettes, seul notre exemplaire II (le plus proche techniquement de I) se prête à une comparaison. A s'en tenir à l'horizontalité du fond, à la hauteur et à la place du pied, seuls l'échantillon isolé du Mont-Beuvray et, parmi les séries associées, celle de Saint-Rémy offrent de bons termes de comparaison (fig. 2,2 et 4). Les exemplaires de Roanne, bien qu'assez proches, ont des proportions un peu différentes (fig. 2,3 ).

Nous voyons ainsi que les ressemblances que l'on observe, à des degrés divers, entre l'écuelle I et le reste du groupe se justifient sans mal, malgré une origine typologique différente. On constate, en effet, ailleurs, d'après des fouilles d'habitats, que ce genre de vase est fabriqué selon les mêmes procédés que des prorluits imitant les formes sigillées. Les limites de cette similitude, toutefois, ont été, selon les sites et l'abondance du matériel, plus ou moins exactement définies d'après l'aspect, largement ou très soigneusement caractérisé, des echantillons. Il'autre part, la typologie des fragments identifiables de Saint-Germain ne permet de comparer ceux-ci qu'aux séries accouplées de Roanne de de Saint-Rémy-rn-Rollat, avee cette particularité que notre assiette diffère de ses drux homologues tantòt par la couleur, tantòt par les proportions du pied et qu'en revanche elle unit une technique de fabrication proche de celle de Roanne à une forme comparable (en ses parties conservies) à celle de Saint-Rémy. Nous voyons, en tout cas, que le matériel de confrontation dont j'ai pu disposer fait ressortir des liens extrêmement probables entre certains de nos tessons el des céramiques du Bassin moyen de la Loire, produites durant la première moitié du $\mathrm{I}^{\mathrm{er}} \mathrm{s}$.

Les vases de la collection Rambert, malgré leur petit nombre et leur pietre ctat de conservation, apportent ainsi d'intéressantes nouveautés. Réputés pour provenir du soussol de Vichy, ils portent des marques inédites ou, du moins, peu connues et que rien ne permet, pour l'heure, de rattacher aux ateliers du Yord et de l'Est de la Gaule ou aux fabriques anglaises. L'hypothèse d'une origine locale de ces produits n'est done pas à rejeter, d'autant moins que l'on possède des preuves, rares encore mais peu contestables, de l'existence, dans le Nord-Est dı Massif Central, d'autres vases imitant la sigillée et apparemment étrangers aussi aux grandes fabriques déja citées. On peut noter, d'autre part, que des signatures attribuables à certains de nos potiers sont peut-être déjà attestées dans la même région et, surtout, que la forme de deux des pièces, dont le profil est en partie déterminé, a des analogues indiscutables découverts dans des habitats ou des sites de production du

58 Op. cil., p. 6.5.

59 op. cil., p. 219 el 223. 

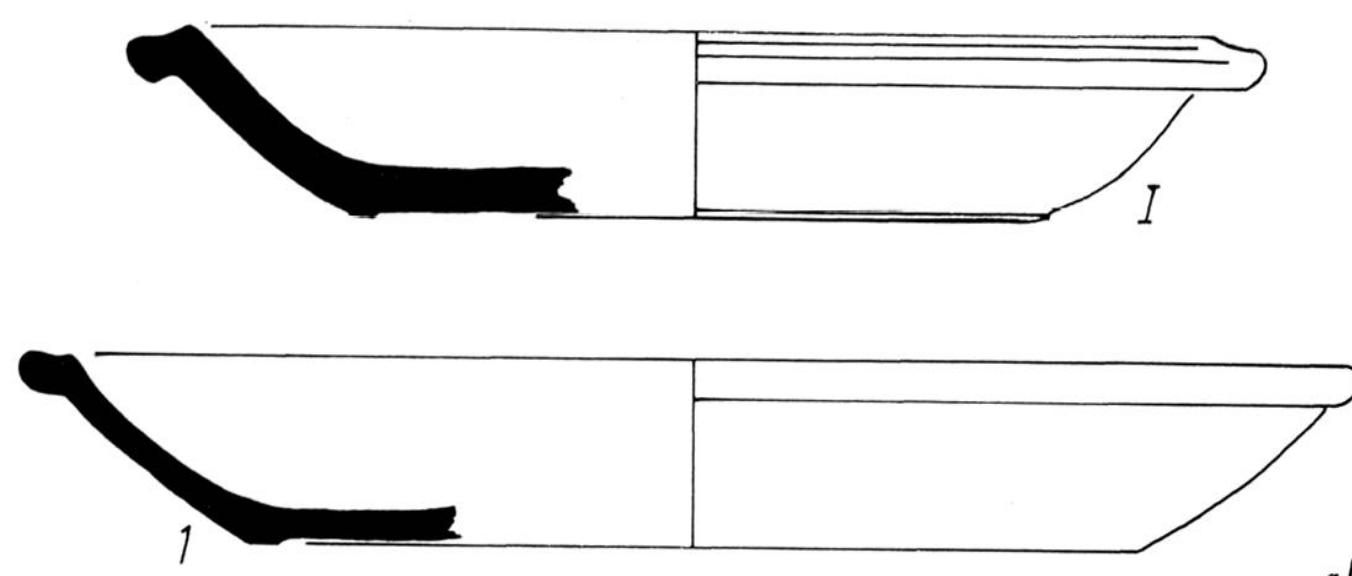

1

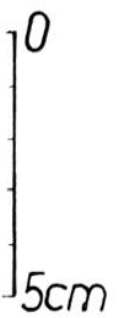

6 Écuelles a live moulurée. I : coll. Rambert ; 1 : exemplaire de Roanne d'apris J. Cabotse et R. Pirichox,

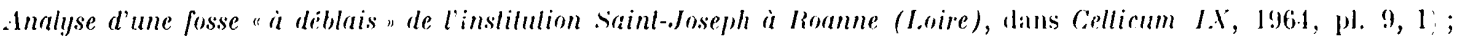

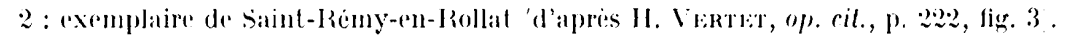

Bassin moyen de la Loire, alors qu'on ne constate de ressemblance aussi caractérisée avec aucun autre matériel publié. Or ces parentés typologiques ne sont pas négligeables, dans des variétés de céramiques communes, auxquelles on ne connaît d'ordinaire qu'une aire de diffusion sans comparaison avec celle des poteries sigillées. Nous disposons ainsi non de preuves, mais d'un faisceau de présomptions, diversement fondées selon l'échantillon et qui plaident en faveur non seulement de l'origine locale de la collection Rambert, mais surtout de la fabricalion a faible distance de Vichy, sinon dans la ville, des vases gris, fins, que l'on y aurait trouvés.

Si nous admettons cette éventualité comme la plus vraisemblable, nous devons admettre, du même coup, que les estampilles, qui fournissent cinq noms d'artisans, témoignent, pour quatre ateliers, au moins, d'une fabrication, dans le Centre, qu'on ne peut placer au delà du premier tiers du I er s. de notre ère. D'autre part, la typologie des vases d'imitation nettement caractérisés par leur forme ou leur signature se trouve étendue pour cette région : parmi les formes identifiées, on ne signalait que des assiettes, deux modèles creux (bols ou bol et jatte ?) -- dont l'un de haute époque - sont maintenant attestés. La connaissance de la céramique hybride d'imitation se trouve done enrichie dans une contréc où sa rareté s'explique, au moins partiellement, par la concurrence précoce des vraies sigillées.

Au dela des simples acquisitions qu'elle peut ainsi apporter. l'étude de cette série d'objets pose quelques problemes. Ln effet les similitudes techniques que, comme d'autres, j'ai observées, amenent, une nouvelle fois, à vérifier que les mèmes modes de fabrication sappliquent à des formes d'origines apparemment différentes. Nous avons, en outre, 
désormais la preuve que ces associations de modeles disparates peuvent englober des vases signés, alors qu'elles n'intéressaient, dans les exemples relevés sur d'autres sites du Cientre, que des pièces d'imitation anépigraphes. I'autre part, les comparaisons typologiques auxquelles conduit cette enquête montrent que l'influence d'une même forme sigillée se retrouve sur des produits de qualités différentes : j'ai exposé ailleurs comment profils et estampilles d'origine italique se retrouvent dans des céramiques grises décorées; nous voyons ici comment la même inspiration transparaît sur des échantillons fumigés - gris ou noirs, engobés ou lissés - et sur des vases non fumigés.

Les observations les plus précises faites jusqu'à présent sur le matériel gallo-romain du Centre ont porté principalement, et avec les meilleures raisons, sur les distinctions techniques entre les différentes céramiques communes. Peut-être ne serait-il pas dénué d'intérêt, pour mesurer plus exactement les aspects que prend localemenl un phénomène, très général, de contamination de traditions diverses, d'envisager systématiquement la fréquence et la régularité de certaines associations typologiques, dans le même centre de production, ou dans la même qualité de fabrication (l'un et l'autre caractères n'étant pas nécessairement liés), et de (hercher dans quelle mesure, selon les cas, la similitude des produits implique un synchronisme étroit des différents types de vases. Peut-être serait-il utile, aussi, d'observer quelles formes techniques diverses, selon les sites, prend l'imitation de modèles sigillés connus et sûrement identifiables ${ }^{60}$ et pendant combien de temps elle persiste.

Colette Bŕnowr.

60 On pourrail ainsi s'interroger sur la place, dans ce groupre des pseudo-sigillées rouges des débuts de l.rzoux

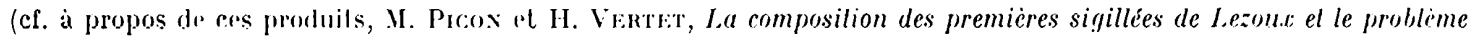
des céramiques catcaires, dans Ret. archéol. Est, XXI, 1970, p. 215-216). 